\title{
Metacognição e seus 50 anos: uma breve história da evolução do conceito
}

\author{
Metacognition and its 50 years: a brief history of the evolution of the \\ concept
}

\section{Metacognición y sus 50 años: breve historia de la evolución del concepto}

Cleci Teresinha Werner da Rosa1; Nancy Nazareth Gatzke Corrêa²; Marinez Meneghello Passos 3 ; Sergio de Mello Arruda ${ }^{4}$

\section{RESUMO}

Os estudos em metacognição avançaram nas últimas décadas, todavia, ainda não há um corpo teórico amplo capaz de abarcar as especificidades possíveis de serem estabelecidas. Essa constatação nos levou a realizar um breve histórico da introdução do termo "metacognição", apresentando uma possibilidade para delimitar sua utilização vinculada às intervenções didáticas no campo educacional. Para isso, texto discute a origem do termo; aponta uma possível instituição de componentes para sua conceitualização a partir de entendimentos consensuais; elabora um mapa dos domínios metacognitivos e assume a reflexão metacognitiva como a expressão que interliga os componentes metacognitivos.

Palavras-chave: Metacognição; Domínios metacognitivos; Experiência metacognitiva.

\begin{abstract}
Studies in metacognition have advanced in recent decades, however, there is still not a broad theoretical body capable of encompassing all the specificities and possible relationships to be established. This finding led us to make a brief history of the introduction of the term "metacognition", presenting a possibility to define its use linked to didactic interventions in the educational field. For this, text discusses the origin of the term; points to a possible institution of components for its conceptualization based on consensual understandings; elaborates a map that represents the metacognitive domains and that assumes the metacognitive reflection as the expression that links the metacognitive components.
\end{abstract}

Keywords: Metacognition; Metacognitive domains; Metacognitive experience.

${ }^{1}$ UPF - Doutora em Educação Científica e Tecnológica pela Universidade Federal de Santa Catarina (UFSC). Docente do Programa de Pós-Graduação em Educação e do Programa de Pós-Graduação em Ensino de Ciências e Matemática da Universidade de Passo Fundo, Passo Fundo/RS - Brasil.

2 UEL - Doutoranda do Programa de Pós-Graduação em Ensino de Ciências e Educação Matemática (PECEM) da Universidade Estadual de Londrina, Londrina/PR - Brasil. Docente da SEED, governo do Estado do Paraná/PR - Brasil.

3 UEL - Doutora em Educação para a Ciência pela Universidade Estadual Paulista "Júlio de Mesquita Filho" (UNESP). Docente Sênior da Universidade Estadual de Londrina, Londrina/PR - Brasil. Docente Sênior colaboradora da Universidade Estadual do Norte do Paraná (UENP), Câmpus Cornélio Procópio, Cornélio Procópio/PR - Brasil.

${ }^{4}$ UEL - Doutor em Educação pela Universidade de São Paulo (USP). Professor Sênior da Universidade Estadual de Londrina, Londrina/PR - Brasil. 


\section{RESUMEN}

Los estudios sobre metacognición han avanzado en las últimas décadas, sin embargo, aún no existe un cuerpo teórico amplio capaz de cubrir las especificidades que se pueden establecer. Esta observación nos llevó a hacer una breve historia de la introducción del término "metacognición", presentando la posibilidad de definir su uso vinculado a intervenciones didácticas en el campo educativo. Para ello, el texto discute el origen del término; apunta a una posible institución de componentes para su conceptualización basada en entendimientos consensuados; elabora un mapa de dominios metacognitivos y asume la reflexión metacognitiva como la expresión que vincula los componentes metacognitivos

Palabras clave: Metacognición; Dominios metacognitivos; Experiencia metacognitiva.

\section{INTRODUÇÃO}

"Pensar sobre o pensar", "cognição da própria cognição", "pensamento do próprio pensamento", podem ser consideradas as expressões mais utilizadas pelos pesquisadores para se referir ao entendimento do que Flavell cunhou nos anos da década de 1970 como sendo "metacognição". De fato, essas expressões representam uma boa aproximação e talvez possam ser representativas do significado do termo, que em 2021 completa 50 anos desde as pesquisas iniciais envolvendo a metamemória.

Entretanto, como menciona Brown (1987), a compreensão de metacognição enquanto processo metacognitivo já estava presente antes de Flavell anunciar o termo. A autora destaca os estudos de Dewey (1910), Thorndike (1917) e Locke e Pringle-Pattison (1924) como aqueles que se referiam ao processo metacognitivo sem, no entanto, utilizá-lo nesses termos. Podemos acrescentar a esses autores Piaget, Vygotsky e até mesmo Sócrates, que, em seus diálogos, instituíram um método de instrução em que o professor respondia a perguntas com novas perguntas. O objetivo do método instituído por Sócrates estava em levar o estudante a perceber suas lacunas de conhecimento para que assim pudesse buscar alternativa para saná-las, uma proposta de tomada de consciência sobre o próprio conhecimento com a consequente ação de regulação em busca da aprendizagem.

Todavia, a forma como discutimos atualmente a metacognição e sua expressividade nos estudos nos campos da Psicologia e da Educação, dentre outros, pode ser datada a partir dos trabalhos do psicólogo americano John Hurley Flavell. Esses estudos, inicialmente relacionados à memória, têm servido para expressar como os seres humanos são capazes de refletir sobre suas experiências cognitivas e, particularmente, no campo educacional, em que medida isso pode lograr êxito nos processos de aprendizagem. Esse entendimento, embora consensual, revelou-se amplo e capaz de possibilitar que as diferentes áreas que se serviram desse conceito fossem agregando novos e distintos elementos, como veremos ao longo deste artigo.

Atualmente podemos dizer que, seja qual for o conceito de metacognição - e suas especificidades adotado nas pesquisas desenvolvidas nas mais diferentes áreas do conhecimento, percebemos que há uma relação com a noção do pensamento sobre o próprio pensamento. Esse pensamento, como expresso por Hacker (1998), pode ser o que alguém sabe (conhecimento metacognitivo), o que alguém está atualmente fazendo (habilidade metacognitiva) ou o estado afetivo ou cognitivo atual de alguém (experiência metacognitiva). Ou, ainda, em uma forma mais generalizada, pode ser entendido como a relação entre a consciência e o controle das ações, em um processo de 
retroalimentação que leva o sujeito a pensar sobre o que sabe (e, alternativamente, sobre o que não sabe), bem como possibilita o controle sobre suas ações, de modo a ser capaz de descrevê-las e avaliá-las frente a uma determinada meta - o que Nelson e Narens (1994) definem como ações de monitoramento e controle.

Essa compreensão, inicialmente apresentada por Flavell, foi sendo ampliada e adaptada ao contexto de diferentes áreas além da Psicologia e da Educação, tornando a metacognição um termo polissêmico e difícil de ser definido. A situação fica ainda mais complexa quando se busca apresentar um conceito suficientemente capaz de abarcar os diferentes campos que têm se utilizado dele em suas múltiplas dimensões.

Veenman, van Hout-Wolters e Afflerbach (2006) ponderam que, em termos da aproximação da metacognição com o campo educacional, é necessário considerar que:

\begin{abstract}
Um grande grupo de pesquisadores está envolvido em determinar as relações complexas entre, de um lado, experiências metacognitivas, crenças epistemológicas, conhecimento metacognitivo e autorregulação, e de outro, processos motivacionais, autoeficácia e interesse (Boekaerts, 1997; Efklides \& Vauras, 1999; Mason \& Scrivani, 2004; Pintrich \& De Groot, 1990; Pintrich \& Schunk, 2002, Zimmerman \& MartinezPons, 1990). Outros estão preocupados com a relação entre metacognição e variáveis afetivas, como a ansiedade (Tobias \& Everson, 1997; Veenman et al., 2000). Alguns se concentram no papel da metacognição em distúrbios ou deficiências de aprendizagem (Borkowski, 1992; Harris, Reed, \& Graham, 2004; Swanson, Christie \& Rubadeau, 1993). (p.10, tradução nossa).
\end{abstract}

Tal gama de possibilidades de utilização do conceito no campo educacional mostra que, embora haja uma polissemia em torno de sua definição, isso não diminui a valorização do seu emprego; ao contrário, mostra a potência desse conceito. Por certo que, se a metacognição representa um modo de pensamento, há múltiplas possibilidades de sua associação a diferentes situações presentes no contexto da aprendizagem, como expresso pelos autores citados.

Sobre o conceito de metacognição, Proust (2013) indica que, embora as áreas discordem quanto ao seu detalhamento e componentes, assim como ao contexto de aplicação e aos métodos, há um consenso no sentido de que a metacognição representa o conhecimento do conhecimento e a regulação da cognição (ou controle executivo e autorregulador), particularmente, quando se trata dos processos educativos.

Frente a essa polissemia, todavia alicerçados nos estudos que mostram os benefícios que a vinculação da metacognição aos processos educativos tem trazido à aprendizagem (HATTIE, 2009; AVARGIL; LAVI; DORI, 2018; VALENZUELA, 2019), ocupamo-nos de resgatar a trajetória histórica de instituição do uso do termo e apresentar uma possibilidade teórica para delimitar sua utilização no âmbito das intervenções didáticas no campo da Educação, bem como elaborar a ideia de um mapa que represente os domínios metacognitivos com ênfase à reflexão metacognitiva.

Assim, o texto não pretende apresentar uma revisão sistemática e crítica da literatura, senão um convite àqueles que buscam entender de que forma a ativação do pensamento metacognitivo tem sido utilizada ao longo dos 50 anos de discussões na interlocução com a aprendizagem e como pode continuar beneficiando esse processo no campo da Educação. 


\section{CONTRIBUIÇÕES DE JOHN FLAVELL E ANN BROWN}

Para discutirmos as bases históricas evolutivas do conceito de metacognição, necessitamos iniciar pelos estudos de Flavell. De acordo com Zohar e Barzilai (2013), mesmo que não haja uma unanimidade em torno do entendimento de metacognição na atualidade, fruto dos múltiplos campos que têm se servido dela, temos uma unanimidade em termos de indicar que a origem do uso do termo metacognição se deve a John Flavell. Paralelamente às discussões de Flavell, temos as contribuições de Ann Brown, que passa a elaborar processos de operacionalização desse conceito e inferir novas discussões sobre sua vinculação com os processos cognitivos. Na continuidade passamos a nos ocupar dos estudos e das inferências desses dois autores.

Hacker (1998) menciona que, dentre as muitas e notáveis realizações de Flavell, precursor do conceito de metacognição, está a utilização do trabalho de Piaget nos Estados Unidos da América. A psicologia desenvolvimentalista de Piaget teve "tremendo impacto" sobre o modo como pesquisadores, médicos e o público (em geral) conceituam o desenvolvimento cognitivo de crianças e de adolescentes. Embora recentes avanços na Psicologia do desenvolvimento tenham indicado necessidade de modificar algum trabalho de Piaget, muitos dos progressos para a instituição do que entendemos hoje como metacognição encontraram ímpeto em suas teorias.

Pautado na perspectiva piagetiana, Flavell analisou, em suas investigações, como as crianças entendiam suas próprias dificuldades em memorizar determinados assuntos e o quanto acreditavam ter memorizado, ressaltando que a memória está intrinsecamente relacionada com os mecanismos que utilizamos para ativá-la e que, a seu ver, são decorrentes da forma como entendemos que esses mecanismos ajudam a memorizar ou a recuperar informações. Lembrar de datas, placas de carro, senhas ou outros dados, a partir de situações que serão facilmente ativadas pelo sujeito, representam ocasiões em que recorremos a um apoio porque estamos cientes de como funciona nossa memória. Esse processo de reconhecer o que é preciso fazer para favorecer o processo de memorização levou Flavell a cunhar o termo "metacognição", como veremos na sequência.

$\mathrm{Na}$ busca por localizar um momento que demarque o início das pesquisas que envolvem metacognição, identificamos certo consenso de que isso se dá a partir do estudo, publicado em 1971 por Flavell, intitulado First discussant's comments: what is memory development the development of? No artigo, o termo utilizado foi "metamemory", ou seja, "metamemória", mas que na continuidade deu origem ao termo "metacognição":

De que é então o desenvolvimento da memória? Parece em grande parte o desenvolvimento de estruturação e armazenamento inteligentes de dados, de operações inteligentes de busca e recuperação e de monitoramento e conhecimento inteligentes dessas operações de armazenamento e recuperação - um tipo de "metamemória", talvez. Essa é a natureza do desenvolvimento da memória. Vamos todos sair e estudá-lo! (FLAVELL, 1971, p. 277, tradução nossa).

O termo "metacognição" aparece no capítulo assinado por Flavell e intitulado Metacognitive aspects of problem solving, o qual integra a obra The nature of intelligence, organizada por Lauren Resnick em 1976. O termo surge no momento em que o autor indaga a si próprio sobre a origem de suas suposições acerca do desenvolvimento da memória:

Minhas próprias suposições sobre o assunto se originam no local esperado, a saber: a área em que fiz a maior parte de minhas pesquisas e pensamentos recentes. Essa área é o desenvolvimento da "metacognição" e, principalmente, de uma subespécie chamada "metamemória" (Kreutzer; Leonard; Flavell, 1975). "Metacognição" refere- 
se ao conhecimento que se tem dos próprios processos e produtos cognitivos ou qualquer assunto relacionado a eles, por exemplo, as propriedades relevantes da aprendizagem de informações ou dados (FLAVELL, 1976, p.232, tradução nossa, assinalamentos do autor).

A partir da publicação desse capítulo, podemos dizer que as pesquisas envolvendo o entendimento cunhado por Flavell e ancorado nos estudos sobre metamemória passam a se difundir, e autores como Brown iniciam aproximações com seus campos de investigação. O próprio Flavell começa uma busca por desenvolver um conceito capaz de atender as pesquisas que estavam sendo desenvolvidas a partir da sua definição primeira, datada de 1976.

No estudo intitulado Metamemory, publicado por Flavell em conjunto com o psicólogo Henry Wellman (1977), encontramos um detalhamento da compreensão de conhecimento metacognitivo, que retomaremos na seção seguinte. No referido artigo, os autores ocupam-se da discussão de questões vinculadas ao desenvolvimento da memória, mencionando que ela não se restringe a trocas de informações, mas contempla as formas de armazenamento e de recuperação dessas informações. $\mathrm{E}$ destacam que, para conhecer o funcionamento da memória de forma mais ampla, podemos estruturála em dimensões cognitivas que de certo modo se sobrepõem. Essas representam a gênese da concepção de metacognição.

A partir dos estudos sobre metamemória, e buscando respostas para questões emergentes no campo da Psicologia do desenvolvimento, Flavell estabelece um modelo que denomina de "Modelo de Monitoração Cognitiva", publicado em 1979. As questões que Flavell buscou explicar por meio desse modelo podem ser resumidas em: Como as informações são armazenadas e recuperadas na estrutura cognitiva? Como essas estruturas se desenvolvem com a idade? Como o armazenamento e a recuperação são controlados?

Além disso, Flavell procurou mostrar, por meio desse modelo, o papel fundamental que a metacognição desempenha na comunicação oral e na aquisição da linguagem, nos processos de leitura e escrita, bem como no auxílio à capacidade do sujeito para resolver problemas e aperfeiçoar a aprendizagem.

O modelo proposto parte do entendimento de que a regulação do pensamento metacognitivo ocorre pela ação e interação de quatro aspectos: conhecimento metacognitivo, experiências metacognitivas, objetivos cognitivos e ações cognitivas. Logo, para que um sujeito ative seu pensamento metacognitivo, quatro aspectos precisam estar conectados, fornecendo os substratos necessários a essa ativação. Nas palavras de Flavell (1979, p. 906-907, tradução nossa):

O conhecimento metacognitivo é aquele segmento de seus conhecimentos de mundo armazenados (quando criança ou adulto), que tem feito as pessoas serem criaturas cognitivas, com suas diversas tarefas, objetivos, ações e experiências. [...] As experiências metacognitivas são quaisquer experiências conscientes cognitivas ou afetivas, que acompanham e pertencem a toda empreitada intelectual. [...] Objetivos (ou tarefas) referem-se aos objetivos do empreendimento cognitivo. As ações (ou estratégias) referem-se às cognições ou a outros comportamentos empregados para consegui-las.

Segundo o autor, os limites que separam os quatro aspectos são tênues, tendo em vista que a metacognição representa a tomada de consciência e a avaliação da cognição. Na continuidade, ele atribui ao processo metacognitivo uma capacidade autorreguladora que age sobre o sistema cognitivo, o que até então tinha um papel secundário em seus estudos. Essa autorregulação decorre 
da identificação pelo sujeito dos seus conhecimentos, tanto em termos do conteúdo específico como de sua capacidade para adquiri-lo, recuperá-lo e manipulá-lo. Tal ampliação mostra que a definição mais adequada para a metacognição, na acepção de Flavell, envolve tanto o conhecimento que o sujeito tem sobre seus conhecimentos (eventos cognitivos) quanto o modo pelo qual ele regula e controla os processos da cognição.

A descrição do modo como poderá ocorrer esse controle inclui planejar, revisar e avaliar o progresso cognitivo. Contudo, a especificação de como isso se traduz em operações de execução pelo sujeito no desenvolvimento de uma atividade específica é mais bem explicitada nos estudos de Brown.

Em suas pesquisas, Brown constata que os alunos com maior facilidade em aprender levantam questões de forma espontânea sobre o tema que estão lendo, o que já não acontece com os que apresentam dificuldade. Os primeiros fazem previsões, refletem se o que estão lendo ou desenvolvendo faz sentido, questionam-se permanentemente sobre suas ações frente à meta estabelecida. Os alunos que têm dificuldade em aprender, no entanto, não aplicam essas estratégias; ao contrário, não questionam ou refletem espontaneamente sobre o que estão fazendo, tornando-se reprodutores de passos previamente estabelecidos.

Tais estudos, desenvolvidos a partir das pesquisas em leitura de textos, levam Brown e seus colaboradores a trabalharem com a hipótese de que alguma estratégia poderia ter vantagens sobre outras. De forma mais específica, Brown (1978) mostra que o procedimento de questionar e refletir, considerado em algum grau como metacognitivo, possibilita que os sujeitos levem vantagem na execução das atividades, como é o caso da leitura de textos. A autora exemplifica mencionando a importância de serem identificados no texto o objetivo e seus aspectos relevantes; de se concentrar a atenção nos tópicos principais; de se avaliar a qualidade da compreensão efetivada; de se verificar se os objetivos estão sendo alcançados; de promover as devidas correções quando existirem falhas na compreensão; de observar o andamento da leitura e corrigi-la quando houver distração, divagações ou interrupções.

Ao focar suas investigações nas atividades de leitura, buscando um método para estimular os aprendizes a ler de forma independente, Brown volta-se à utilização dos mecanismos autorregulatórios que ocorrem durante a realização de uma determinada ação ou tarefa e que, no entender de Flavell, representa o segundo componente metacognitivo - o controle executivo e autorregulador. No artigo intitulado Metacognition, executive control, self-regulation, and other more mysterious mechanism, Brown esclarece que a metacognição "refere-se à compreensão do conhecimento, uma compreensão que pode ser refletida no uso efetivo ou na descrição aberta do próprio conhecimento" (Brown, 1987, p. 65, tradução nossa).

Em tal artigo, a autora detalha os aspectos que pertencem ao mecanismo de controle executivo e autorregulador. Em suas palavras:

O segundo grupo de atividades, que é chamado de metacognitivo na literatura sobre desenvolvimento, consiste nas atividades usadas para regular e supervisionar o aprendizado. Esses processos incluem atividades de planejamento (previsão de resultados, estratégias de agendamento e várias formas de tentativa e erro indiretos etc.). Antes de iniciar um problema, monitorar atividades (monitorar, testar, revisar e reprogramar as estratégias de aprendizado) durante o aprendizado; e verificação de resultados (avaliação do resultado de qualquer ação estratégica com base em critérios de eficiência e eficácia) (BROWN, 1987, p. 68, tradução nossa). 
Esse grupo de operações, que será detalhado na próxima seção, tem servido para orientar a ativação do pensamento metacognitivo não apenas no contexto das estratégias de leitura, mas também no campo da resolução de problemas (MALONE, 2008; TAASOOBSHIRAZI; FARLEY, 2013) e outras atividades vinculadas ao contexto escolar.

Outra contribuição apresentada por Brown e colaboradores para ampliar as discussões inicialmente apresentadas por Flavell, diz respeito ao processo de automatização do pensamento metacognitivo nos sujeitos, na forma de "piloto automático". Brown (1987) mostra que a utilização desse pensamento pode se tornar automática nos sujeitos com o passar do tempo. É o que acontece no caso dos leitores que aplicam estratégias metacognitivas, mesmo sem se darem conta de que o fazem, tendo em vista que o seu uso é automático.

Esse automatismo leva a outro aspecto apontado por Brown, relativo ao fato de a metacognição se encontrar no plano da consciência ou no inconsciente ${ }^{5}$ do sujeito. A exemplo de Flavell, ela assume que a metacognição se refere ao controle consciente e deliberado das próprias ações cognitivas, distinguindo a cognição inconsciente e automática da metacognição consciente e deliberada (BROWN, 1978). Posteriormente, Brown, Campione e Day (1981) reconhecem a existência de processos cognitivos inferiores da consciência e que se caracterizam pela rapidez e pelo automatismo, sem, por isso, deixarem de ser processos inteligentes, relacionando-os à metacognição. No artigo de 1987, ela permanece com a definição de metacognição como algo relacionado ao plano da consciência, mas adverte que se trata de tema complexo e difícil de ser esclarecido.

Todavia, Martinez (2006, p. 698, tradução nossa), ao refletir sobre o fato de a metacognição ser um processo automatizado, destaca que:

A metacognição às vezes pode ter a qualidade da automação. Isso ficou claro para mim um dia enquanto eu estava dando uma aula. Uma aluna observou uma contradição em algo que eu disse durante uma palestra e levantou a mão quase reflexivamente - sem nenhum pensamento consciente anterior, segundo o que ela me disse mais tarde. Talvez esse tipo de automação seja o que imaginamos ter uma pessoa reflexiva que desenvolveu hábitos críticos da mente. A metacognição pode se tornar tão praticada, tão normal, que, como um hábito mental, pode adquirir o status de traço de personalidade.

Embora existam discussões sobre esse tema, e ainda que se trate de um processo consciente, encontramos dificuldades para esclarecê-lo. Todavia, isso não tem se traduzido em empecilho ao avanço nas pesquisas em metacognição, principalmente aquelas vinculadas às práticas de intervenção pedagógica no campo educacional. Para exemplificar, citamos o estudo de Zohar e Barzilai (2013), que, após realizarem uma revisão de estudos sobre metacognição no campo da Educação em Ciências, revelam que o tema vem crescendo em interesse no cenário nacional, particularmente a partir dos anos 2000. A revisão de Corrêa et al. (2020) para o uso do termo "metacognição" também revela que, no Brasil, as pesquisas nesse mesmo campo, incluindo a Matemática, têm crescido significativamente. Tal situação é igualmente verificada na revisão realizada por Rosa e Schmitz (2020) para o campo da Educação, ao mostrarem que o número de estudos cresce no País, particularmente os relacionados à aprendizagem em Matemática, assim como os que envolvem expertise e sua vinculação com o pensamento metacognitivo.

\footnotetext{
${ }^{5} \mathrm{O}$ sentido de inconsciente atribuído por Brown refere-se ao nível mais baixo de consciência ou subconsciente.
} 
As contribuições de Brown e Flavell para a instituição da metacognição como um campo profícuo de investigações representam os aspectos iniciais na discussão sobre a importância desse tipo de reflexão de pensamento por parte dos sujeitos para o desenvolvimento cognitivo. Além disso, representam as discussões introdutórias sobre a ideia de a metacognição ser um processo que se torna automatizado nas ações dos sujeitos, ou mesmo se ela se encontra no nível da consciência ou nos planos mais inferiores dessa consciência. A partir desses estudos, autores têm se debruçado na perspectiva de esmiuçar esse debate, investindo na busca por consolidar a metacognição como uma estratégia de ensino e de aprendizagem que pode contribuir consideravelmente na qualificação da aprendizagem.

Dessa forma, ao finalizar a seção, ressaltamos que o apresentado mostra que o termo "metacognição" pode ser historicamente atribuído ao psicólogo americano John Hurley Flavell. Todavia, autores como Ann Lesley Brown contribuíram para ampliar as discussões e a compreensão em torno desse construto. A diversidade de áreas que passaram a se servir das discussões iniciais de Flavell sobre metacognição, mesmo que restrinjamos o olhar apenas ao educacional, evidencia possibilidades para a instituição de uma "teoria metacognitiva", que ainda carece de discussões e convergências para se estabelecer como tal.

Apesar de essa teoria ainda não estar estabelecida, é preciso registrar que a metacognição apresenta alguns aspectos que poderiam dar sustentação a essa busca e que têm se revelado predominantes, não únicos, nas pesquisas, como o fato de que ela está assentada em dois componentes, um referente à consciência dos processos cognitivos (conhecimento metacognitivo) e, o outro, à autorregulação e ao gerenciamento desses processos em relação à tarefa de aprendizagem (habilidades metacognitivas). Essa compreensão compartilhada por Flavell e Brown encontra na atualidade sua identificação, como proposto por Veenman, Van Hout-Wolters e Afferbach (2006). Podemos acrescentar a esse rol as experiências metacognitivas, que, embora não apareçam como um componente no entendimento desses autores, recebem atenção especial, pois são coadjuvantes aos conhecimentos metacognitivos. Ou seja, podemos entender que, para uma situação ser representativa do domínio metacognitivo, necessitaria envolver os três aspectos mencionados.

\section{EXPERIÊNCIA, CONHECIMENTO E HABILIDADE METACOGNITIVA}

Como evidenciado anteriormente, a experiência, o conhecimento e as habilidades metacognitivas utilizando-se termos empregados por Veenman, van Hout-Wolters e Afflerbach (2006) - assumem relevância em uma interpretação sobre a metacognição, particularmente em se tratando de sua relação com a aprendizagem, como mostrou Flavell. Embora já tenhamos falado desses componentes na seção anterior, julgamos pertinente retomá-los, agora com o objetivo de especificá-los, possibilitando uma compreensão a partir da interpretação para além das expressas por Flavell e Brown.

Vamos iniciar pela discussão do entendimento de experiências metacognitivas, cuja compreensão em Flavell (1979, p. 906-907, tradução nossa) foi de que elas são dadas por "quaisquer experiências conscientes cognitivas ou afetivas, que acompanham e pertencem a toda empreitada intelectual".

Anastasia Efklides tem dedicado grande parte de suas pesquisas a discutir o significado das experiências metacognitivas, mostrando que elas são as desencadeadoras do conhecimento metacognitivo e compreendem os sentimentos de motivação, confiança, contentamento, estímulos, 
entre outros, empregados com intuito de recuperar informações na estrutura cognitiva ou mesmo de controlar a ação. A autora chama a atenção para o fato de que a experiência metacognitiva representa algo mais do que uma emoção ou afeto, estando relacionada a julgamentos, sentimentos ou reações expressas pelas pessoas e associadas ao desenvolvimento de uma atividade, que pode ser uma tarefa ou a resolução de um problema (algo mais complexo que uma tarefa).

A experiência metacognitiva é apresentada por Efklides (2001) como sendo a experiência que a pessoa vivencia durante um esforço cognitivo, abrangendo o conhecimento metacognitivo on-line, ideias, crenças, sentimentos, objetivos e julgamentos, ou seja, o conhecimento envolvido pelo sentimento presente na memória de curto prazo, a própria experiência subjetiva. Este produto do monitoramento on-line não contempla apenas as características da tarefa, incluem também as preocupações e os objetivos da pessoa em relação à tarefa, sendo influenciada por fatores referentes: à tarefa (complexidade, desempenho e experiências anteriores com tarefas relacionadas); à pessoa (capacidade cognitiva, personalidade e autoconceito); e a fatores metacognitivos (conhecimento metacognitivo).

Efklides (2009) afirma que as experiências metacognitivas, enquanto manifestações do monitoramento on-line da cognição durante a realização de uma atividade que exige um esforço cognitivo compreendem: sentimentos metacognitivos, estimativas metacognitivas, juízo de memória e conhecimentos específicos da tarefa online. Complementando, Efklides (2014) esclarece que o controle consciente no nível da consciência pessoal, acionado pela experiência metacognitiva, pode abranger o conhecimento geral da pessoa, o afeto, a percepção do contexto da tarefa que envolve manifestações ativadas pelo contato com o mundo, como também afirmam Corrêa, Passos e Arruda (2018a, 2018b); e a metacognição social, que contém manifestações ativadas pelo contato com o outro, também citados por Corrêa, Passos e Arruda (2018a, 2018b).

As experiências metacognitivas ocorrem em fases distintas. Ao se deparar com uma atividade, antes de iniciá-la, na fase prospectiva, segundo Efklides (2006a), as manifestações são: sentimento de familiaridade, sentimento de saber, sentimento de dificuldade, estimativa de solução correta ou estimativa de tempo de resolução. Durante a realização da atividade, as manifestações presentes são: sentimento de dificuldade, estimativa de esforço, estimativa de tempo necessário para completar a tarefa, elaboração de estratégias a serem usadas. E ao finalizar a atividade na fase retrospectiva as manifestações são: estimativa de correção da solução, sentimento de confiança, sentimento de satisfação, sentimento de dificuldade, além de outras respostas emocionais.

Buscando compreender melhor as manifestações da experiência metacognitiva, serão apresentados aspectos e características das manifestações da experiência metacognitiva, que se fazem presentes nas pesquisas sobre esse domínio metacognitivo.

Os 'sentimentos' enquanto manifestações da experiência metacognitiva abrangem: sensação de saber, sentimento de familiaridade, sentimento de dificuldade, sentimento de confiança e sentimento de satisfação. Estes sentimentos informam a pessoa sobre aspectos muito específicos do processamento cognitivo, contudo, o fazem de maneira experiencial, que, segundo Frijda (1986), tem natureza hedônica. Estando muitas vezes um correlacionado ao outro, por exemplo, o sentimento de dificuldade e a estimativa de esforço, ou a sensação de familiaridade e o sentimento de confiança, entre outros. 
As 'estimativas' ou julgamentos metacognitivos, segundo Efklides (2009), podem ser resultado do inconsciente que informa conscientemente a seleção, aplicação e controle das estratégias, compreendendo: estimativa de aprendizagem (envolvendo o sentir e conhecer fenômenos), solução exata (influenciada pela crença na capacidade cognitiva, autoconceito e autoeficácia), empreendimento de esforço (relacionado ao sentimento de facilidade ou dificuldade), estimativa de tempo (influenciada pela complexidade ou demanda da tarefa).

O 'juízo de memória', ou monitoramento da metamemória, inclui a memória declarativa e processual, refere-se ao que o sujeito entende por características da memória universal, intrapessoal ou interpessoal, que não se limitem à cognição como: origem da memória, lembrança ou precisão, tratado por Nelson (1993), como julgamento de aprendizagem ou, segundo Johnson, Hashtroudi e Lindsay (1993), "quando" e "como" a informação foi adquirida. A frequência ou recência da informação, que segundo Efklides, Samara e Petropoulou (1999) está relacionada ao monitoramento da metamemória; e a memória episódica (relacionada com a consciência autonoética6 ${ }^{6}$.

Outra forma de experiência metacognitiva, de acordo com Flavell (1979), é o conhecimento metacognitivo on-line, que monitora os recursos da tarefa atendidos e mantidos na memória de curto prazo, como também as operações cognitivas executadas na entrada da tarefa para a execução dos objetivos estabelecidos pelo sujeito que realiza a tarefa, expresso como "conhecimento específico da tarefa on-line' (EFKLIDES, 2001).

O 'contexto' foi abordado por Efklides (2001) e Corrêa, Passos e Arruda (2018a, 2018b), para as manifestações que acompanham o fato ou a situação responsável por acionar a conscientização, sendo uma manifestação da experiência metacognitiva por incitar um processo reflexivo metacognitivo, podendo este ser um livro, um texto, um vídeo, uma ocorrência em sala de aula ou no processo de interação com o mundo (objetos e lugares), que não se restringiram aos aspectos cognitivos, mas que incentivaram processos reflexivos acionando a consciência de conhecimentos prévios que influenciam a autoavaliação da cognição e a autocompreensão, ou, ainda, a necessidade de fazer escolhas ou tomar decisões. Processos que envolvem incertezas são processos que podem acionar manifestações da experiência metacognitiva, segundo Paris (1999).

Os 'sujeitos' também podem acionar a consciência por meio de manifestações da experiência metacognitiva, que acontece no contato com o outro. Paris (1999) afirma que a metacognição pode se originar das próprias pessoas ou de outras, por exemplo, quando outra pessoa questiona por que o outro fez algo ou como realizou determinada tarefa, ou ainda na autoapresentação, quando é desejado que o sujeito tenha determinados pensamentos sobre o outro. Corrêa, Passos e Arruda (2018a, 2018b) afirmam que as manifestações da experiência metacognitiva podem ocorrer na realização de comparações cognitivas que acontecem nas relações sociais de convívio (interpessoal), relacionadas ao autoconhecimento e ao autoconceito, que segundo Efklides $(2001,2014)$ e Flavell (1979) são características pessoais que influenciam as experiências metacognitivas.

Em linhas gerais, Efklides (2006b) mostra que as experiências metacognitivas são aquelas que levam o sujeito a dar continuidade ou interromper um processo de pensamento, uma ação de natureza cognitiva. Isso estaria relacionado à identificação pelo sujeito de suas capacidades (e conhecimentos)

${ }^{6}$ Consciência autonoética, segundo Weeler, Stuss e Tulving (1997), é entendida como a consciência de autoconhecimento, referindo-se à capacidade do sujeito de representar mentalmente e tornar-se consciente de suas experiências subjetivas no passado e no presente, e de suas projeções no futuro, uma integração de informações sobre si e suas experiências. 
para executar uma ação, possibilitando, ainda, avaliar se o desenvolvimento poderá lograr êxito frente à meta estabelecida.

Nessa compreensão, as experiências metacognitivas podem ser entendidas como propulsoras da ativação do conhecimento metacognitivo e das habilidades metacognitivas (controle executivo e autorregulador). Diferentemente de Flavell e Wellman (1977), Efklides entende que as variáveis pessoa, tarefa e estratégia - estariam vinculadas às experiências e deveriam ser avaliadas pelo sujeito antes da tomada de decisão sobre a realização de uma tarefa ou resolução de um problema. Para Flavell e Wellman (1977), tais variáveis são integrantes do conhecimento metacognitivo, como veremos na continuidade.

O conhecimento metacognitivo representa o conhecimento que um indivíduo tem sobre seu conhecimento e o uso que faz dele para aprender como realizar a busca no mundo externo, baseado em uma recuperação interior de como realizar esse jogo entre o que "está dentro" e o que "está fora". Flavell e Wellman (1977, p. 5, tradução nossa), referindo-se à memória, inferem que essa recuperação ou armazenamento vincula-se a variáveis que integram os processos cognitivos do sujeito:

[...] o desempenho em uma situação de memória ou tarefa é influenciado por um número de fatores cuja natureza uma pessoa deveria saber. Nós temos três classes principais de tais fatores [variáveis]: (1) características relevantes de memória da própria pessoa [variável pessoa]; (2) características relevantes de memória para a tarefa [variável tarefa]; (3) estratégias potenciais de emprego/uso [variável estratégia].

A variável "pessoa" representa os atributos pessoais temporários e duradouros que são relevantes para a recuperação de informações. Ela está vinculada ao autorreconhecimento como um ser mnemônico em relação aos outros, ou seja, à consciência das próprias habilidades em comparação às do outro enquanto ser cognitivo; à percepção das próprias capacidades e limitações, que se diferenciam das que outros apresentam. Isso acontece quando o sujeito constata, por exemplo, que é bom em reconhecer números e datas, mas julga não ter a mesma facilidade para lembrar nomes de pessoas.

Flavell e Wellman (1977) chamam atenção para o fato de que esse conhecimento das próprias habilidades pode ser algo vindo da experiência vivenciada pelos sujeitos, que pode nos dizer e nos ensinar em quais aspectos podemos obter maior potencialidade ou, ao contrário, em quais temos maior dificuldade. De certa forma é possível entender que as experiências vivenciadas se relacionam com a capacidade de autoconhecimento e das características pessoais.

Flavell (1979) aponta que a variável pessoa pode estar relacionada ao caráter: universal, em que o sujeito tem crenças a respeito do funcionamento da mente humana; intraindividual, referenciando características do sujeito com relação à sua própria aprendizagem, como sobre aprender melhor ouvindo que lendo; e interindividual, referindo-se às características de aprendizagens presentes nos outros sujeitos, por exemplo, que algum amigo é mais apto a aprender determinado conceito que ele.

A segunda variável, "tarefa", está relacionada à demanda e às exigências que promovem a ativação ou recuperação da memória. Rosa (2011, p. 45) mostra que o que está em jogo nessa variável é a "identificação pelos sujeitos das características da tarefa em pauta, tanto em termos do que ela é como do que envolve". 
Para Flavell e Wellman (1977), podemos ter um conhecimento considerável quando o mesmo vem por meio da aquisição a partir de propriedades diferentes das informações que podem ser caracterizadas como unidades individuais de informações, isoladas com relação a outras. As unidades que são facilmente codificadas (rotuladas, imaginadas), podem apresentar-se mais significativas e familiares ou mais fáceis que outras.

A maioria das propriedades relevantes de recuperação deve estar relacionada com essas unidades e também com a estrutura e a organização de todos os subconjuntos de unidades de informação. Uma parte dessa informação pode ativar facilmente outras, se ela trouxer elementos da variedade da forma. Essa relação se dá de duas maneiras na experiência: sendo parte de um grupo ou de um todo; ou relatado por meio de conexões lógicas ou relações causais. As relações significativas podem fazer uma sugestão de recuperação para outra, e aprendizes mais maduros têm consciência disso. As pessoas podem adquirir a capacidade, a habilidade de fazer julgamento a respeito da complexidade de uma tarefa, estimando a influência de uma propriedade em relação a outra. Outras habilidades que o aprendiz mais experiente pode apresentar é a capacidade de reconhecer quais são as propriedades relevantes e não relevantes no que diz respeito à recuperação de uma informação ou ativação de um pensamento (Flavell \& Wellman, 1977).

A respeito da variável "estratégia", Flavell e Wellman (1977) estabelecem relação com a forma pela qual identificamos os mecanismos que são necessários ativar para recuperar algo na memória. Segundo Rosa (2011, p. 46), esse é o "momento em que o sujeito se questiona sobre o que precisa ser feito e quais os caminhos a serem seguidos para atingir o objetivo".

A variável "estratégia" envolve o conhecimento do dossiê de táticas de resolução, procedimentos, aplicabilidade e eficácia com relação tanto à demanda da atividade a ser realizada como do contexto ao qual ela está inserida, com a finalidade de realizar a atividade com êxito.

Há uma variedade de movimentos específicos que podem servir para isso, mas as experiências em relação a outras ações ou outros processos de recuperação de informações são essenciais e se revelam fundamentais para o êxito. Quando identificamos o que precisamos fazer, como precisamos fazer ou de que forma devemos proceder, podemos estar nos referindo a um pensamento metacognitivo que foi ativado em função de situações experienciadas que nos permitiram chegar a ele. Essa variável é uma espécie de função executiva que dá início a essa busca na estratégia, usando um processo de controle para dar conta das demandas da tarefa.

Para Flavell, Miller e Miller (1999), esta variável está relacionada à identificação dos sujeitos de quais estratégias são mais adequadas para chegar a determinados resultados cognitivos. Elas estão relacionadas às experiências e se constituem por meio delas. Ao vivenciar situações, armazenamos informações e sentimentos que nos permitem subsidiar ações futuras e proceder com escolhas pautadas por aquilo que experienciamos.

Por fim, mencionamos que, de acordo com Flavell e Wellman (1977), as variáveis apresentadas precisam atuar juntas, influenciando-se mutuamente, no sentido de auxiliar a ativação do pensamento metacognitivo. Portanto, em ações que buscam a tomada de consciência sobre os próprios conhecimentos, é necessário oportunizar a ativação dessas três variáveis como forma de ser mais eficiente nessa atividade.

Autores como Schraw e Dennison (1994) especificam essas variáveis em termos dos conhecimentos, estabelecendo uma relação com o conhecimento declarativo, que se refere à descrição de fatos e 
conceitos e ao conhecimento de argumentos lógicos de raciocínio, que pode geralmente dar explicações científicas sobre o tema; o conhecimento processual, apoiado na perspectiva da execução (ou não) das tarefas solicitadas; o conhecimento condicional, associado ao conhecimento que temos do porquê pensamos frequentemente a partir das relações "se" e "então". Trata-se de uma questão de estruturação do entendimento de como a tomada de consciência metacognitiva pode ser elaborada, embora ela possa estar vinculada com o especificado por Flavell e Wellman (1977).

Pode-se entender o conhecimento declarativo com referência às variáveis: pessoa, tarefa e estratégia. O conhecimento declarativo pessoal está associado a um conhecimento de longo prazo, que envolve autoconhecimento e autoconsciência incluindo crenças sobre o pensamento, diz respeito ao que sabe, não sabe e precisa saber para realizar uma determinada atividade, segundo Kluwe (1982); para a variável tarefa, entende-se que a reflexão promove a consciência da complexidade, extensão, organização, particularidades ou especificidades daquela atividade a ser realizada; e com relação à estratégia pode-se entender que depende da compreensão, reflexão e conscientização da importância da transferibilidade de táticas entre tarefas e contextos.

O conhecimento processual refere-se ao conhecimento de processos e ações que Schraw (1998) denomina de conhecimento metaestratégico, ao relacionar com as variáveis: pessoa, tarefa e estratégia. Pode-se compreender o autoconceito e autoeficácia, com relação à variável pessoa, podendo afetar a seleção, aplicação ou transferência de estratégias na realização de uma atividade; com relação à variável tarefa refere-se ao conhecimento dos objetivos e da natureza da tarefa, podendo ser influenciada pelas crenças de dificuldade de realização; e com relação à variável estratégia, a sensibilidade da tarefa influencia a identificação de estratégias apropriadas e aplicáveis.

O conhecimento condicional envolve "quando" e "por que" usar o conhecimento declarativo e processual, podendo ser inibido por conhecimentos inadequados de conceitos, fraco monitoramento cognitivo e ineficiência na utilização de estratégias.

No que diz respeito às habilidades metacognitivas, que estão associadas ao que Flavell denominou de "controle executivo e autorregulador" (como registrado anteriormente) e que foram detalhadas por Brown, trazem a evidenciação de três elementos: planificação, monitoramento e avaliação.

A 'planificação' implica na previsão de etapas e seleção de estratégias a partir da identificação da meta desejada frente à atividade. Para Brown (1987), o planejamento é possível a partir do momento em que o sujeito conhece o problema ou a atividade proposta. Segue a autora esclarecendo que, quanto melhor o sujeito conhece o problema, melhor e de forma mais eficiente ele pode planejar suas ações. Rosa (2011) evidencia que, em termos da aprendizagem escolar, a planificação está associada à identificação, por exemplo, da organização de materiais para estudar ou à estruturação de um questionário referente ao tema em estudo com objetivo de guiar o estudante.

Efklides (2009), ao detalhar os tipos de estratégias envolvidas nas habilidades metacognitivas, sinaliza que estratégias de orientação têm por objetivo tornar explícitas as exigências da tarefa, e o planejamento em si envolve a elaboração de uma sequência de tentativas de processos de ordem superior que controlam os processos necessários para o processamento da tarefa, como o estabelecimento de objetivos e a sequência de procedimentos, cronogramas e pontos de verificação para monitorar o progresso da realização da atividade.

O 'monitoramento', por sua vez, refere-se à possibilidade que o sujeito tem de compreender e modificar uma ação durante a sua execução, reavaliando decisões e planejamentos. Na 
aprendizagem, Rosa (2011) relata que a interpretação de Brown pode ser visualizada no momento em que o aluno procede com uma revisão dos conhecimentos no instante em que está construindo os novos, de modo a poder avaliar se está ou não no caminho certo para atingir o objetivo da construção do conhecimento.

Para o monitoramento, Efklides (2009) aponta que a regulação do processo cognitivo acontece quando o processamento inicial não está ocorrendo satisfatoriamente, envolvendo o acompanhamento da efetivação da sequência de processos planejados, como atrasos na execução do cronograma, além da detecção de erros e discrepâncias entre ação e plano, verificação da aplicação apropriada de estratégias ou uso de instrumentos ou ferramentas, envolvendo a identificação de novas necessidades que surgem da implementação dos novos planos.

Finalmente, a 'avaliação' está vinculada aos resultados atingidos frente ao objetivo traçado no início da atividade ou ação, podendo, eventualmente, ser especificada pelos critérios exclusivos de avaliação. No âmbito escolar, Rosa (2011, p. 56) observa que "esse é o momento em que os estudantes retomam e avaliam a aprendizagem com o intuito de identificar como realizaram". Esse momento pode servir para entender o processo de execução da atividade, o conhecimento dela decorrente, ou, ainda, para identificar possíveis falhas no processo.

As estratégias de avaliação para Veenman e Elshout (1999) envolvem a avaliação do resultado do processamento cognitivo em relação aos critérios ou aos padrões previamente estabelecidos, podendo envolver estratégias para a avaliação da qualidade do planejamento, regulação e implementação das estratégias usadas para monitorar o processamento das tarefas. Enquanto Schunck e Zimmerman (1998) sinalizam que no processo de avaliação são utilizadas estratégias de recapitulação e autorregulação, envolvendo estratégias para a avaliação de todo o empreendimento com uma tarefa, do que aconteceu do começo ao fim do processamento, dos pontos fortes e fracos, das causas dos resultados e do que deve ser atendido no futuro.

Veenman e Elshout (1999) apontam que as habilidades metacognitivas referem-se ao uso deliberado de estratégias para controlar a cognição, envolvendo a atenção seletiva da memória de trabalho, que engloba: estratégias de orientação, estratégias de planejamento, estratégias de regulação do processamento cognitivo, estratégias para monitorar a execução da ação planejada e estratégias para a avaliação do resultado do processamento da tarefa.

Diante desse trajeto delineado, em que retomamos a origem do termo "metacognição" e alguns desdobramentos teóricos e práticos relacionados a ele no desenvolvimento de pesquisas e aplicações durante 50 anos, encerramos este artigo inserindo avanços interpretativos teóricos e que estão pautando o desenvolvimento das investigações realizadas por nosso grupo de pesquisa quanto à observação dos processos metacognitivos em sala de aula.

\section{CONSIDERAÇÕES CONCLUSIVAS: MAPEANDO OS DOMÍNIOS METACOGNITIVOS}

Partindo dos conceitos apresentados, nas seções anteriores deste artigo, foi realizada uma sistematização contemplando três elementos representativos dos domínios da metacognição conhecimentos metacognitivos, experiências metacognitivas, habilidades metacognitivas - buscando assim esclarecer e possibilitar a visualização dos conceitos e respectivos entrelaçamentos. 
Nesse momento entende-se que o construto "metacognição" apresenta-se multifacetado, como afirma Efklides (2006b, 2009), e sistêmico, pois ao acionar um dos domínios da metacognição, inevitavelmente, os outros entram em ação de forma inter-relacional, por meio de uma complexa e elaborada rede reflexiva que atua no sistema cognitivo/emocional e influencia, profundamente, os processos de aprendizagem, conectando-se à compreensão do conhecimento dos processos internos e externos, envolvendo a cognição e os sentimentos (processos de interpretação das emoções), por meio dos processos de autoconhecimento e da autorregulação, acionados pela reflexão metacognitiva a partir do contato com o outro, com o mundo e consigo mesmo.

Na Figura 1, procuramos representar essas diversas facetas, dando a elas uma ordenação e coerência, a fim de gerar um mapa que possa orientar as investigações em sala de aula.

Como podemos observar agilmente no centro deste mapa (Figura 1), trazemos 'reflexão metacognitiva', assumindo-a como a palavra que interliga os 'conhecimentos metacognitivos', as 'habilidades metacognitivas' e as 'experiências metacognitivas'. Justificamos tal elaboração pelo fato de compreendermos que a estrutura da metacognição é uma estrutura reflexiva, que se retroalimenta à medida que o conhecimento metacognitivo vai sendo adquirido por meio da aplicação das habilidades metacognitivas ou das manifestações da experiência metacognitiva. Também é fato que esses três 'domínios', além de influenciarem, são influenciados pela reflexão metacognitiva na conscientização por meio do conhecimento metacognitivo que passa a ser implementado a partir das experiências metacognitivas, as quais, por sua vez, são ativadas por meio do acesso aos conhecimentos metacognitivos e dos processos autorregulados, durante as aplicações das habilidades metacognitivas. Ou seja, o fato de a 'reflexão metacognitiva' estar inter-relacionando os três domínios da metacognição por meio de setas bidirecionais, sinaliza a natureza sistêmica da conexão entre a reflexão e os domínios metacognitivos. As setas simples procuram mostrar o multifacetamento e a integração desses 'elementos' que assumimos por representativos dos domínios da metacognição e que, atualmente, estão sendo considerados para a coleta e a interpretação de dados de situações que se passaram em sala de aula.

Figura 1 - Domínios da Metacognição

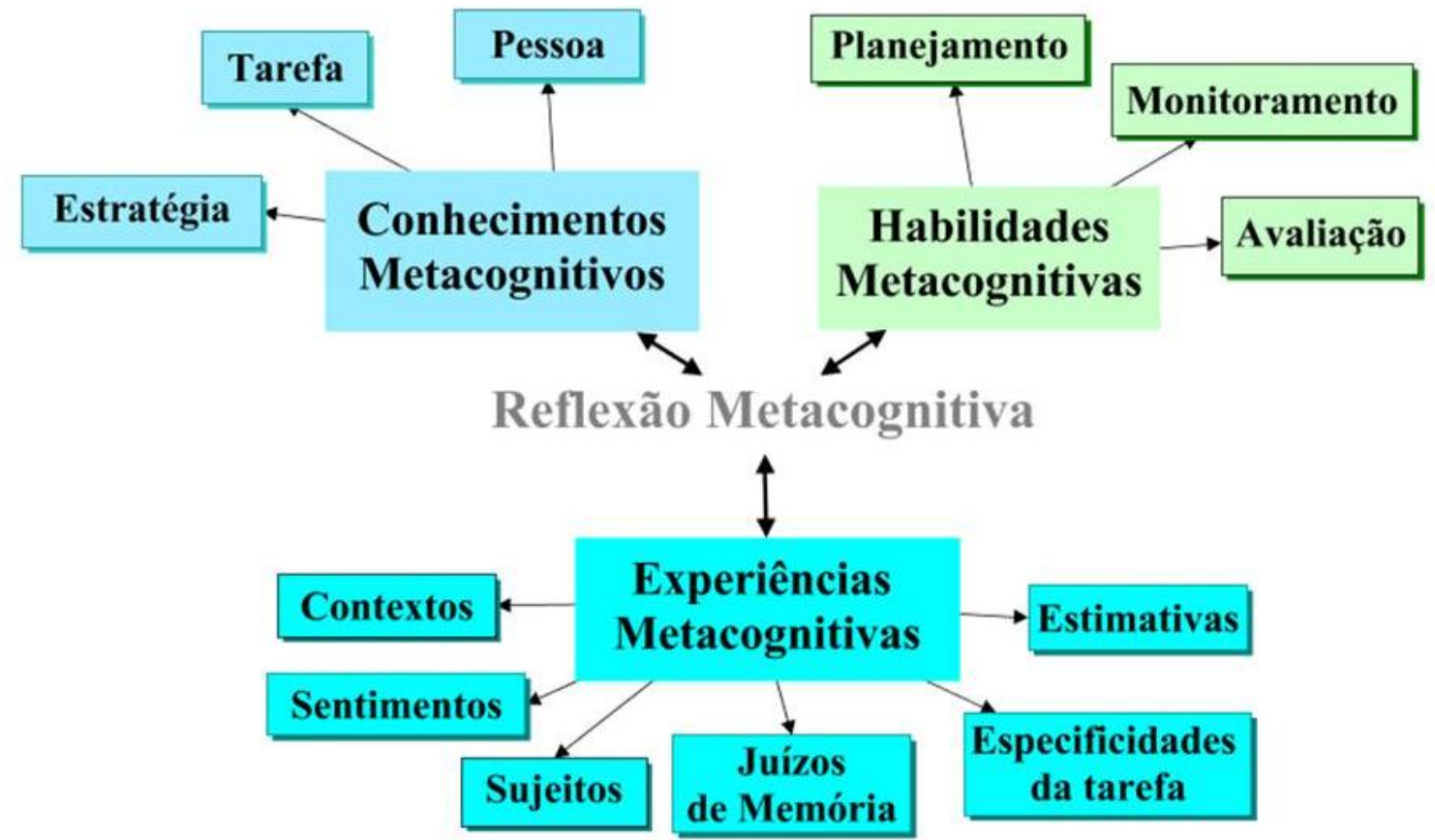

Fonte: os autores 
Complementamos ainda que a reflexão metacognitiva, segundo Cornoldi (1998), não se refere a qualquer processo trivial de reflexão, envolve crenças, conhecimentos prévios, suposições, desafios, capacidades, contextos, percepções, entendimentos de problemas, tarefas ou qualquer atividade cognitiva envolvendo conhecimentos de estratégias e processos, sendo a reflexão essencial para processos metacognitivos, promovendo assim o autoconhecimento que também é identificado como a base para a metacognição.

Cabe também destacar que o domínio do conhecimento metacognitivo está diretamente relacionado às variáveis: pessoa, tarefa e estratégia, variáveis apresentadas inicialmente por Flavell (1979) e exploradas por outros pesquisadores; o domínio das habilidades metacognitivas está conectado ao planejamento, monitoramento e avaliação, também relacionado às premissas de Flavell (1979), quando apresentou as metas/tarefas e as ações/estratégias, que foram ampliadas primeiramente por Brown (1987) e, posteriormente, por outros pesquisadores tanto da área da Psicologia cognitiva como da área educacional; e, por fim, o domínio das experiências metacognitivas que está conectado aos: contextos, sentimentos, sujeitos, juízos de memória, especificidades da tarefa e estimativas, exploradas, essencialmente, por Efklides a partir das definições de Flavell (1979).

Após elucidar os conceitos que estão intrinsecamente conectados aos domínios metacognitivos, sistematizados no mapa e explicitados ao longo do texto, é necessário revalidar as definições iniciais em que a metacognição se apresenta como compreensão da natureza do aprender e do conhecer, em que um sujeito cognitivo só alcança um nível superior de compreensão e autoconhecimento se acionar os dispositivos reflexivos dos domínios cognitivos, os quais se inter-relacionam em uma rede de processos intrínsecos, integrando-se, reorganizando-se e retroalimentando-se de forma consciente ou inconsciente.

\section{REFERÊNCIAS}

AVARGIL, Shirly; LAVI, Rea; DORI, Yehudit Judy. Students' metacognition and metacognitive strategies in science education. In: DORI, Yehudit Judy; MEVARECH, Zemira; BAKER, Dale. Cognition, Metacognition, and Culture in STEM Education. Cham, Switzerland: Springer, 2018. p. 33-64 https://doi.org/10.1007/978-3-319-66659-4

BROWN, Ann L. Knowing when, where, and how to remember; a problem of metacognition. In: GLASER, R. (Ed.). Advances in instructional psychology, Hillsdale, New Jersey: Lawrence Erlbaum Associates, 1978. p. 77-165

BROWN, Ann L. Metacognition, executive control, self-regulation, and other more mysterious mechanisms. In: WEINERT, Franz E.; KLUWE, Rainer H. (Eds.). Metacognition, motivation and understanding. Hillsdale, New Jersey: Lawrence Erlbaum Associates, 1987. p. 65-116.

BROWN, Ann L.; CAMPIONE, Joseph C.; DAY, Jeanne D. Learning to learn: on training students to learn from texts. Educational Researcher, v. 10, p. 14-21, 1981.https://doi.org/10.3102/0013189X010002014

CÔRREA, Nancy N. G.; PASSOS, Marinez M.; ARRUDA, Sérgio M. Metacognição e as relações com o saber. Ciência \& Educação, v. 24, n. 2, p. 517-534, 2018a. https://doi.org/10.1590/1516731320180020016 
CÔRREA, Nancy N. G.; PASSOS, Marinez M.; ARRUDA, Sérgio M. Perfil metacognitivo (Parte II): aplicação de instrumento de análise. Investigações em Ensino de Ciências, v. 23, n. 1, p. 176191, 2018b. http://dx.doi.org/10.22600/1518-8795.ienci2018v23n1p230

CÔRREA, Nancy N. G.; PASSOS, Marinez M.; ARRUDA, Sérgio M. Estudo exploratório sobre o uso da palavra "metacognição" em artigos publicados em periódicos brasileiros do ensino de ciências e matemática de 2007 a 2017. Caderno Brasileiro de Ensino de Física, v. 37, n. 1, p. 6-26, 2020. https://doi.org/10.5007/2175-7941.2020v37n1p6

CORNOLDI, Cesare. The impact of metacognitive reflection on cognitive control. In: MAZZONI, Giuliana; NELSON, Thomas (Eds.). Metacognition and cognitive neuropsychology: monitoring and control processes. Hillsdale, New Jersey: Lawrence Erlbaum Associates, 1998. p. 139-160

DEWEY, John. How we think. Boston. MA: DC Heath, 1910.

EFKLIDES, Anastsy. Metacognitive experiences in problem solving. In: EFKLIDES, Anastsy; KUHL, J.; SORRENTINO, R. M. (Eds.). Trends and prospects in motivation research, Springer, Dordrecht, 2001. p. 297-323 https://doi.org/10.1007/0-306-47676-2

EFKLIDES, A. The systemic nature of metacognitive experiences. In: CHAMBRES, P.; IZAUTE, M.; MARESCAUX, P. J. (Eds.). Metacognition. Springer, Boston, MA., 2002. p. 19-34. https://doi.org/10.1007/978-1-4615-1099-4

EFKLIDES, A. (2006a). Metacognition and affect: What can metacognitive experiences tell us about the learning process?. Educational Research Review, v. 1, n. 1, p. 3-14, 2006a.

https://doi.org/10.1016/j.edurev.2005.11.001

EFKLIDES, Anastasia. Metacognitive experiences: the missing link in the self-regulated learning process. Educational Psychology Review, v. 18, n. 3, p. 287-291, 2006b.

https://doi.org/10.1007/s10648-006-9021-4

EFKLIDES, Anastasia. The role of metacognitive experiences in the learning process. Psicothema, $\mathrm{v}$. 21, n. 1, p. 76-82, 2009.

EFKLIDES, Anastasia; SAMARA, A.; PETROPOULOU, M. Feeling of difficulty: an aspect of monitoring that influences control. European Journal of Psychology of Education, v. 14 n. 4, p. 461-476, 1999. https://doi.org/10.1007/BF03172973

FLAVELL, John Hurley. First discussant's comments: what is memory development the development of? Human Development, n. 14, p. 272-278, 1971. https://doi.org/10.1159/000271221

FLAVELL, John Hurley. Metacognitive aspects of problem solving. In: RESNICK, Lauren B. (Ed.). The nature of intelligence. Hillsdale, New Jersey: Lawrence Erlbaum Associates, 1976. p. 231236.

FLAVELL, John Hurley. Metacognition and cognitive monitoring: a new area of cognitive developmental inquiry. American Psychologist, v. 34, n. 10, p. 906-911, 1979. http://dx.doi.org/10.1037/0003-066X.34.10.906

FLAVELL, John Hurley; WELLMAN, Henry M. Metamemory. In: KAIL, Robert V.; HAGEN, John W. (Eds.). Perspectives on the development of memory and cognition. Hillsdale, New Jersey: Lawrence Erlbaum Associates, 1977. p. 3-33.

FLAVELL, John Hurley; MILLER, P.; MILLER, S. A. Desenvolvimento cognitivo. Tradução de Cláudia Dornelles. 3. ed. Porto Alegre: Artes Médicas Sul, 1999. 
FRIJDA, Nico H. The emotions. Cambridge University Press, 1986.

HACKER, Douglas J. Definitions and empirical foundations. In: HACKER, Douglas J.; DUNLOSKY, John; GRAESSER, Arthur. Metacognition in educational theory and practice. Mahwah, New Jersey: Lawrence Erlbaum Associates, 1998. p. 1-23.

HATTIE, John. Visible learning: a synthesis of over 800 meta-analyses relating to achievement. NY: Routledge, 2009.

JOHNSON, Marcia K.; HASHTROUDI, Sahin; LINDSAY, Stephen. Source monitoring. Psychological Bulletin, v. 114, n. 1, p. 3-28, 1993.

KLUWE, Rainer H. Cognitive knowledge and executive control: metacognition. In: GRIFFIN, D. R., Animal Mind - Human Mind. New York: Springer, 1982. p. 201-224

LOCKE, John; PRINGLE-PATTISON, Andrew Seth. An Essay Concerning Human

Understanding... Abridged and Edited by AS Pringle Pattison. Clarendon Press, 1924.

MALONE, Kathy L. Correlations among knowledge structures, force concept inventory, and problemsolving behaviors. Physical Review Special Topics - Physics Education Research, v. 4, n. 2, p. 020107-15, 2008. https://doi.org/10.1103/PhysRevSTPER.4.020107

MARTINEZ, Michael E. What is metacognition?. Phi Delta Kappan, v. 87, n. 9, p. 696-699, 2006. https://doi.org/10.1177/003172170608700916

NELSON, Thomas 0 . Judgments of learning and the allocation of study time. Journal of Experimental Psychology, v. 122, n. 2, p. 269-273, 1993.

NELSON, Thomas O.; NARENS, Louis. Why investigate metacognition. Metacognition: knowing about knowing, v. 13, p. 1-25, 1994.

PARIS, S. When are metacognition formative, debilitating or benign. In: COLLOQUE METACOGNITION: PROCESS, FUNCTION AND USE. Actes, 10, Clermond-Ferrand: Université Blaiser-Pascal. 1999.

PROUST, Joëlle. The philosophy of metacognition: mental agency and self-awareness. OUP Oxford, 2013.

ROSA, Cleci Teresinha Werner da. A metacognição e as atividades experimentais no ensino de Física. 2011. Tese (doutorado) - Programa de Pós-Graduação em Educação Científica e Tecnológica. Universidade Federal de Santa Catarina, Florianópolis: 2011.

ROSA, Cleci T. Werner da; SCHMITZ, Kymberli. A metacognição nas pesquisas em educação: uma revisão a partir das teses e dissertações brasileiras. Actio: Docência em Ciências, v. 6, n. 2, 2020.

SCHRAW, Gregory. Promoting general metacognitive awareness. Instructional Science, v. 26, n. 1-2, p. 113-125, 1998. https://doi.org/10.1023/A:1003044231033

SCHRAW, Gregory; DENNISON, Rayne Sperling. Assessing metacognitive awareness.

Contemporary Educational Psychology, v. 19, n. 4, p. 460-475, 1994.

https://doi.org/10.1006/ceps.1994.1033

SCHUNK, Dale H.; ZIMMERMAN, Barry J. (Ed.). Self-regulated learning: from teaching to selfreflective practice. New York: Guilford Press, 1998. 
TAASOOBSHIRAZI, Gita; FARLEY, John. A multivariate model of physics problem solving. Learning and Individual Differences, v. 24, p. 53-62, 2013. https://doi.org/10.1016/j.lindif.2012.05.001

THORNDIKE, Edward. L. Reading as reasoning: a study of mistakes in paragraph reading. Journal of Educational Psychology, v. 8, n. 6, p. 323-332, 1917.

VENEZUELA M., Ángel. ¿Qué hay de nuevo en la metacognición? Una revisión del concepto y su aplicación en los procesos de lectura y escritura.2019. Venezuela. Recuperado de https://www.researchgate.net/publication/306079274

VEENMAN, Marcel V. J.; ELSHOUT, Jan J. Changes in the relation between cognitive and metacognitive skills during the acquisition of expertise. European Journal of Psychology of Education, v. 14, n. 4, p. 509-523, 1999. https://doi.org/10.1007/BF03172976

VEENMAN, Marcel V. J.; VAN HOUT-WOLTERS, Bernadette H. A. M.; AFFLERBACH, Peter. Metacognition and learning: Conceptual and methodological considerations. Metacognition and Learning, v. 1, n. 1, p. 3-14, 2006. https://doi.org/10.1007/s11409-006-6893-0

WHEELER, Mark A.; STUSS, Donald T.; TULVING, Endel. Toward a theory of episodic memory: the frontal lobes and autonoetic consciousness. Psychological Bulletin, v. 121, n. 3, p. 331-354, 1997. https://doi.org/10.1037/0033-2909.121.3.331

ZOHAR, Anat; BARZILAI, Sarit. A review of research on metacognition in science education: current and future directions. Studies in Science Education, v. 49, n. 2, p. 121-169, 2013. https://doi.org/10.1080/03057267.2013.847261

Submissão: 21/09/2020

Aceito: 22/10/2020 